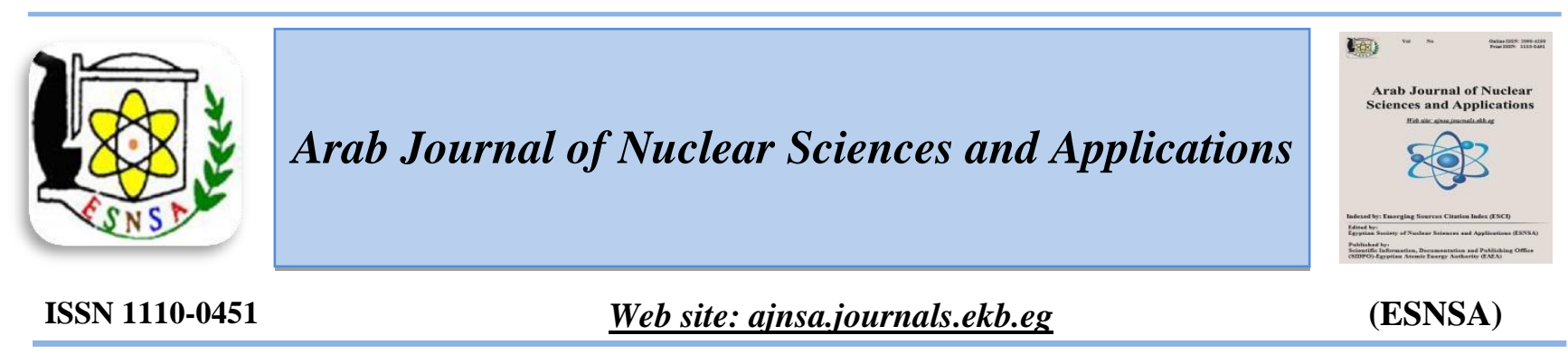

\title{
Long-Term and Flares Variability of Fermi LAT FSRQ 4C + 21.35
}

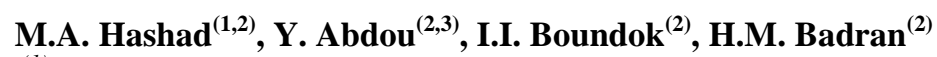 \\ ${ }^{(1)}$ Basic Science Department, Modern Academy for Engineering and Technology, Maadi, Egypt \\ ${ }^{(2)}$ Physics Department, Faculty of Science, Tanta University, Tanta, Egypt \\ ${ }^{(3)}$ Center for Fundamental Physics, Zewail City of Science and Technology, Sheikh Zayed, Giza, Egypt
}

Received $5^{\text {th }}$ Apr. 2018 Accepted $24^{\text {th }}$ Feb. 2019

\begin{abstract}
The current study presents a long-term light curve (LC) of the flat spectrum radio quasar (FSRQ) 4C +21.35 (also known as PKS 1222+216, $\mathrm{z}=\mathbf{0 . 4 3 2}$ ) as observed by the Fermi Large Area Telescope (LAT) during the first seven years of the mission with weekly time binning in the energy range $0.2-50 \mathrm{GeV}$. The highest weekly integral flux reached $2.647 \times 10^{-6} \mathrm{phcm}^{-2} \mathrm{~s}^{-1}$ on MJD $=55365$ (2010 June 18). 4C $+\mathbf{2 1 . 3 5}$ exhibited during this period two major flares (flare I during MJD $=\mathbf{5 5 2 9 6}-\mathbf{5 5 4 0 5}$ and flare II during MJD = 56961 - 56984). For flare states, the variability of the source emission was studied with 12 hour time binning in two energy bands $0.2-0.8 \mathrm{GeV}$ and $0.8-50 \mathrm{GeV}$. Three different statistical methods, the structure functions (SF), auto-correlation functions (ACF), and fast Fourier transformer (FFT) were used to study the time scales. For the seven year-weekly binning light curve, an 18-week time scale was observed. Flare I seems to have the same time scales for both energy ranges. Therefore, the outbursts during flare I may have come from the same emission mechanism/s; CCF between the two energy bands also emphasized it. Flare II did not give significant results. CCF illustrated a 6.5-day time lag that indicates the emission mechanism of the low energy band came before the high energy one with 6.5 day with a typical physical process.
\end{abstract}

Keywords: Non-thermal,FSRQ: Individual 4C+21.35, Galaxies: Active, Galaxies: Statistics

\section{Introduction}

Radio-loud active galactic nuclei (AGN) with relativistic jets oriented at small viewing angles called 'blazars' - are known for their particularly intense and dramatically variable $\gamma$-ray emission within the $\mathrm{GeV}$ photon energy range [1]. Blazars are further classified as flat-spectrum radio quasars (FSRQs) and BL Lacertae objects (BL Lacs) based upon their appearance in the optical-ultraviolet bands. FSRQs have strong broad emission lines in the optical-ultraviolet bands while BL Lacs at most show weak emission lines, sometimes displaying absorption features, and in some cases, can be completely featureless [2]. The radiative output of high luminosity blazars, particularly the GeV $\gamma$-ray FSRQs, is generally explained as a result of Inverse Compton (IC) scattering of photons external to the jet by nonthermal electrons which commonly referred to as External Compton (EC) [3]. A gamma-ray flare was detected in April 2009 from $4 \mathrm{C}+21.35$ [4], one of the most variable and brightest FSRQ in Fermi LAT $3^{\text {rd }}$ catalog. It was followed in December 2009 by an even larger flare, seen by both the AGILE Gamma-ray Imaging Detector and the Fermi LAT. In April 2010, 24 Fermi LAT detected a mostly strong GeV outburst from it [5-7]. The source went again to a quiet state until it underwent the next flare in November 2014 which lasted nearly for one month; see Table (1) for the source parameters.

In the present study, the temporal evolution of $4 \mathrm{C}$ +21.35 in $\gamma$-rays, as observed in the first seven years of the Fermi operation by LAT, was studied

Corresponding author: mhashad64@yahoo.com

DOI: 10.21608 /ajnsa.2019.3402.1078

(C) Scientific Information, Documentation and Publishing Office (SIDPO)-EAEA 
and a detailed statistical analysis of variability during the brightest $\gamma$-ray flares epochs of $4 \mathrm{C}$ +21.35 was also presented. The paper is organized as follows: In Section II, Fermi-LAT observations and data reduction are described. Time analysis methods of variability are presented in Section III. The main results obtained from the LAT analysis with its implications on blazar emission and underlying physical processes are being discussed in section IV. Finally, in Section V findings of the study are summarized.

\section{Data Analysis and Reduction}

The Fermi-LAT is a pair-conversion $\gamma$-ray telescope sensitive to photon energies greater than $20 \mathrm{MeV}$. In its nominal scanning mode4, it surveys the whole sky every three hours with a field of view of about $2.4 \mathrm{sr}$ what's sr [8]. The gamma-ray data used in this work was collected during the first seven years of the nominal all-sky survey by Fermi-LAT, during the time period from August 4, 2008 to August 4, 2015 (MJD 54682 - 57238) with the energy band $0.2-50 \mathrm{GeV}$ as shown in Table (2). The LAT data was analyzed utilizing an unbinned likelihood analysis method using the standard analysis of the Fermi-LAT Science Tools software package [8]. The $\gamma$-ray light curve (LC) was calculated using Fermi Science Tools v10r0p5 and Pass 8 reconstruction algorithms with instrument response function (IRF) P8R2_SOURCE_V6, Galactic diffuse background gll_iem_v06, and isotropic background iso_P8R2_SOURCE_V6_v06. To reject atmospheric $\gamma$-rays from the Earth's limb, events were selected with zenith angles $<100^{\circ} .4 \mathrm{C}+21.35$ and the contaminating sources within $10^{\circ}$ from its sky position were modeled by using a simple power law $\mathrm{F}(\mathrm{E}) \propto \mathrm{E}-\alpha$, where $\alpha$ is the photon index. The flux, photon index, and test statistic (TS) were calculated for each time bin using the maximum-likelihood algorithm implemented in gtlike. The threshold of the Test Statistic for a meaningful detection was set to $9(\sim 3 \sigma)$. The magnificent Enrico package5 was used to run the Fermi Science Tools $[9,10]$.

\section{Time Analysis}

The characterization of the variability properties of AGN puts constraints on its physical conditions and can be used to study the separate structures that compose it. Generally, focus is made on a deterministic signal that is contaminated by random fluctuations. The use of time-series analysis can quantitatively extract information by observation of AGN variability. Several functions such as structure function $(S F)$, autocorrelation function $(A C F)$ and Fast Fourier Transformation (FFT) were used to study the LC variability. Each function has its own advantages [11-16].

\section{Results and Discussion}

The weekly-binned seven-year light curve of $4 \mathrm{C}$ +21.35 , from MJD $=$ for $\gamma$-ray energy from 0.2 to $50 \mathrm{GeV}$ is shown in Figure (1). While no periodic behavior is obviously present, there are some basic features that allow the characterization of the variability. The two largest flux variations in LC were observed between April - July 2010 (Flare I) and Nov. 2014 (Flare II), see Table (3). The $S F$, $A C F$, and FFT of the whole LC for the whole energy range $(0.2-50 \mathrm{GeV})$ are all shown in Figure (2), binned in seven-day intervals.

\begin{tabular}{|c|c|c|c|c|c|c|c|c|}
\hline $\begin{array}{l}\text { Fermi } \\
\text { name }\end{array}$ & $\begin{array}{c}\text { Counterpart } \\
\text { name }\end{array}$ & RA & DEC & $\begin{array}{c}\text { Objet } \\
\text { type }\end{array}$ & $\begin{array}{l}\text { Velocity } \\
(\mathrm{Km} / \mathrm{s})\end{array}$ & $\begin{array}{c}\text { Supermassive } \\
\text { black hole } \\
\text { mass } \mathrm{M}_{\mathrm{BH}}\end{array}$ & $\begin{array}{c}\text { Eddington } \\
\text { luminosity } \\
\mathrm{L}_{\mathrm{Edd}}\end{array}$ & $\begin{array}{c}\text { Redshift } \\
(\mathrm{z})\end{array}$ \\
\hline $\begin{array}{c}4 \mathrm{C} \\
+21.35\end{array}$ & $\begin{array}{c}\mathrm{PG} \\
1222+216\end{array}$ & $12 \mathrm{~h} 24 \mathrm{~m} 54.4 \mathrm{~s}$ & $+21 \mathrm{~d} 22 \mathrm{~m} 46 \mathrm{~s}$ & QSO & $>30000$ & $\begin{array}{c}\simeq 1.5 \times 10^{8} \\
\mathrm{M}_{\odot}\end{array}$ & $2 \times 10^{46} \mathrm{erg}$ & 0.43200 \\
\hline
\end{tabular}

Table (2): Selections Applied for The Download Data

\begin{tabular}{cc}
\hline Parameter in the query & Value \\
\hline Time period & MET $^{\text {a) }}: 239557417-460395817$ \\
& MJD $^{\text {b) }}: 54682.655-57238.655$ \\
Radius & August 4, 2008 to August 4, 2015 \\
Energy range & 20 degrees \\
LAT data type & $200 \mathrm{MeV}-50 \mathrm{GeV}$ \\
Photon
\end{tabular}


Both $S F$ and $A C F$ should not exhibit any variation with time lag and be consistent with horizontal lines when the flux of the source is not experiencing from any significant variation, on average. $A C F$ peaks around 18, 191, and 230 weeks, meanwhile $S F$ shows minima exactly at the same time scales observed in $A C F$ and FFT illustrates time scale around 18 weeks. The 18week time scale seems to be a resultant from variability of flare I as shown in Figure (1).

Table (3) 4C +21.35 maximum values of average flux for weekly time binning for the entire first seven years of LAT observation with the whole energy range and 12-hour time binning with the two energy bands E1 and E2 for both Flare I

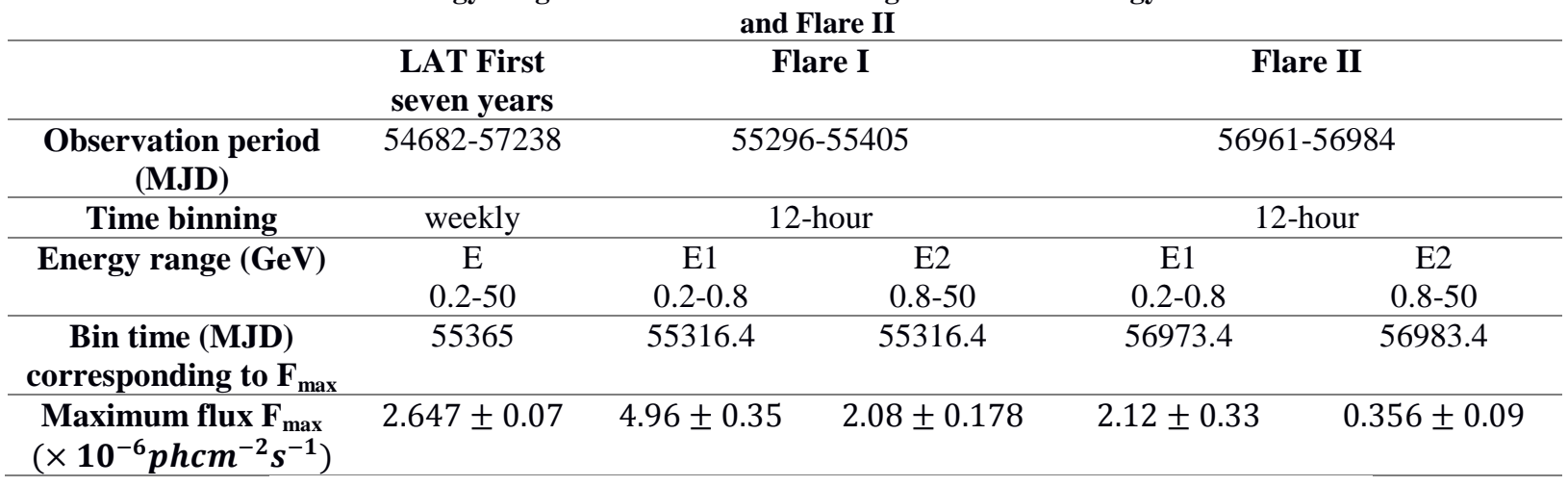

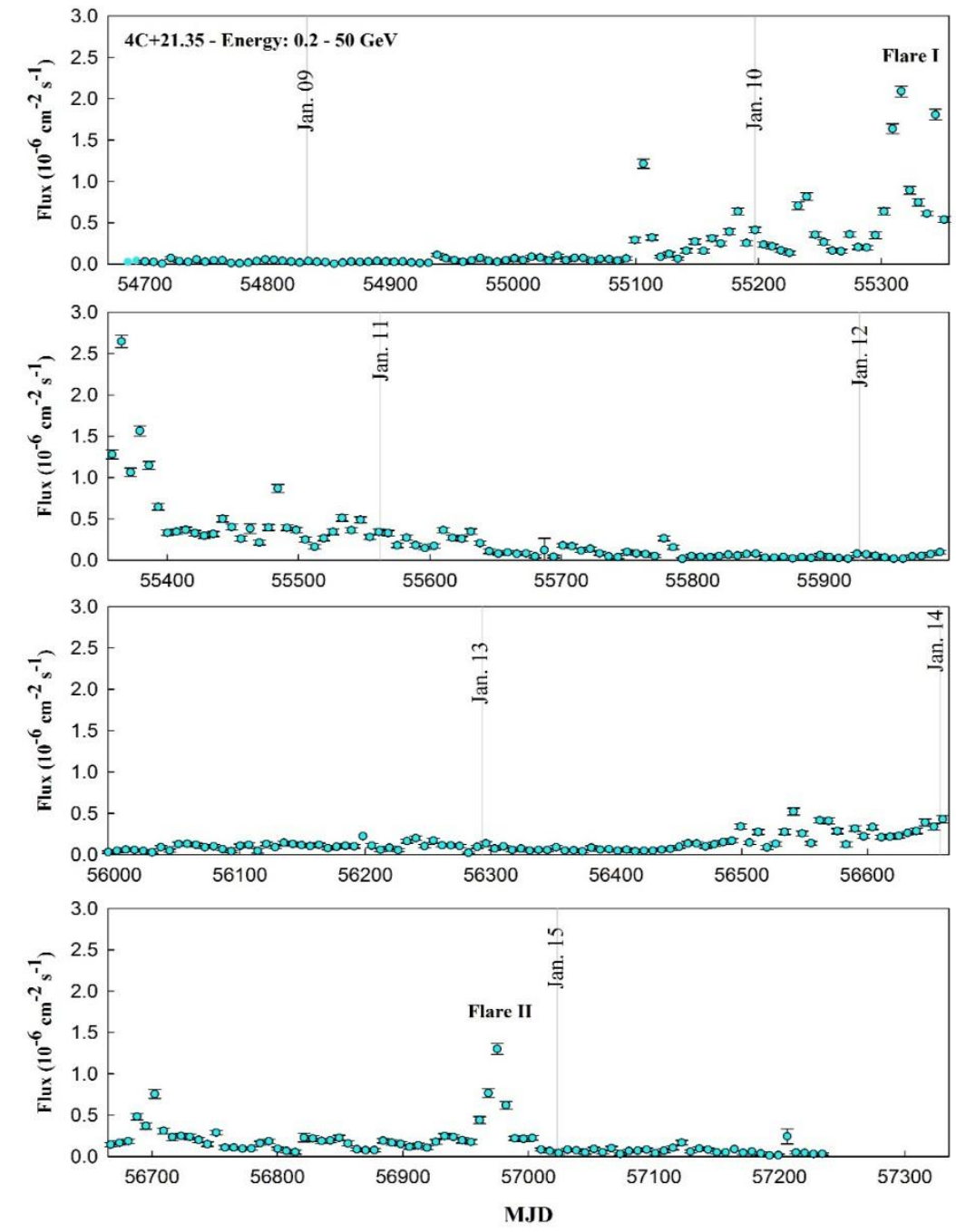

Figure (1) High energy gamma-ray light curve of the blazar $4 \mathrm{C}+21.35$ in the energy range from 0.2 to $50 \mathrm{GeV}$ for seven years of Fermi-LAT data within a 1-week time bin 

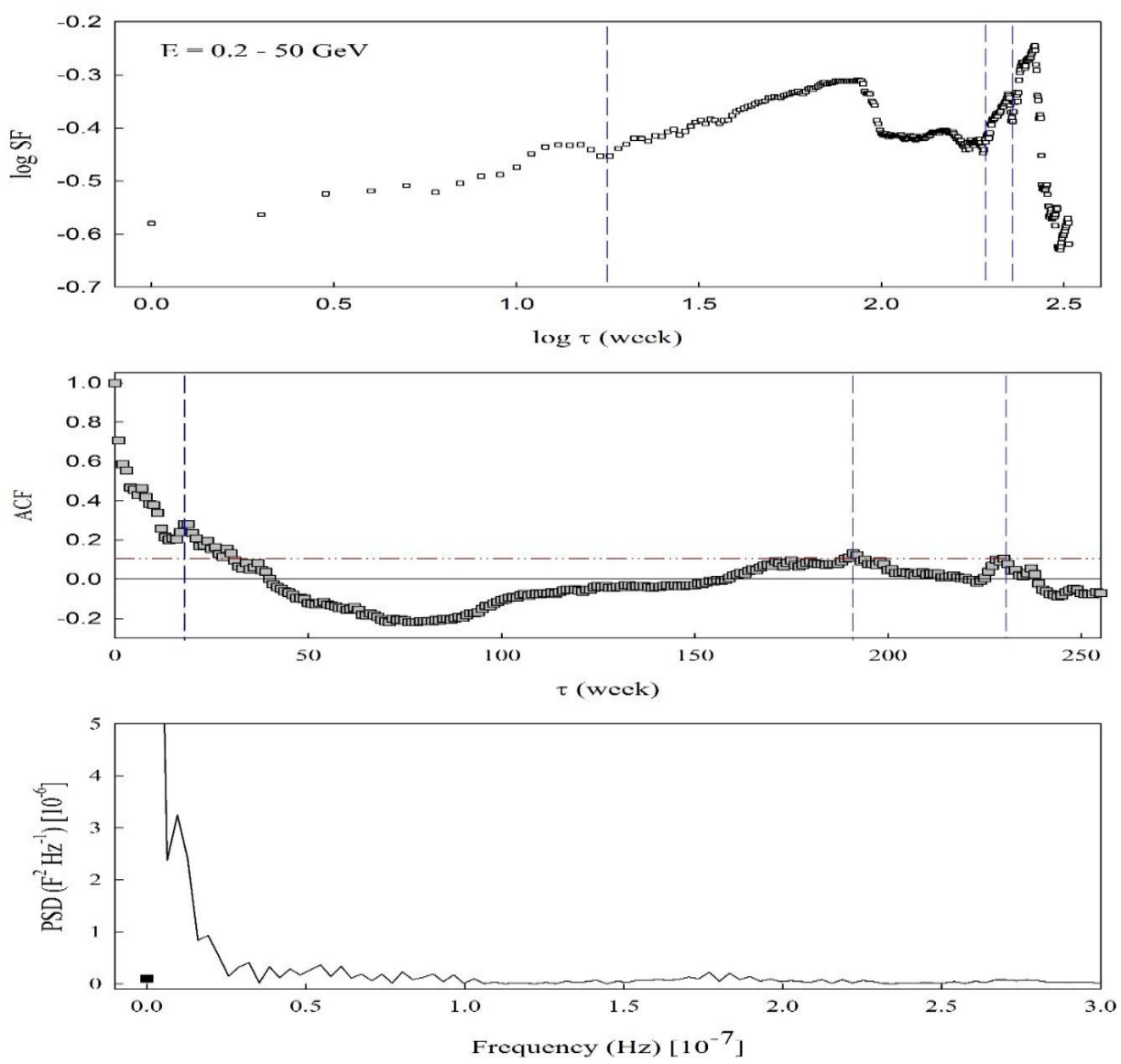

Figure (2) SF, ACF, and PSD of the whole light curve for $200 \mathrm{MeV}-50 \mathrm{GeV}$ gamma-ray energy. Top, middle, and bottom panels are SF, ACF, and FFT, respectively. In middle ACF panel, the dashed dotted horizontal line refers to the confidence level

However, during the flares, the $\gamma$-ray fluxes are calculated using time bin siz e of 12 hours with two energy ranges, $\mathrm{E} 1$ from 0.2 to $0.8 \mathrm{GeV}$ and E2 from 0.8 to $50 \mathrm{GeV}$ as showed in Figure (3). Flare I for the two energy ranges from MJD $=55296$ (2010 Apr. 04) to MJD= 55405 (2010 Jul. 28), see Figure (3). As shown in Table (3), it reached the highest 12-hour integral flux $(\mathrm{E}=0.2-0.8 \mathrm{GeV}) 4.96 \pm 0.35 \times$ $10^{-6} \mathrm{phcm}^{-2} \mathrm{~s}^{-1}$ on MJD $=55316.4$ (Apr. 30, 2010). The 12-hour binning LC of Flare I creates a pattern of repetitive spikes around MJD = 55310, 55326, 55342, 55359, 55365, 55377, and 55386. Figure 3 shows also the LCs of Flare II of the two energy ranges from MJD $=56961$ (2014 Oct. 31) to MJD = 56984 (2014 Nov. 23). It reached the highest 12-hour integral flux $(\mathrm{E}=0.2-0.8 \mathrm{GeV}) 2.12 \pm 0.33 \times$ $10^{-6} \mathrm{phcm}^{-2} \mathrm{~s}^{-1}$ on MJD $=56973.4$ (Nov. 14, 2014). In flare II, for 12 hour binning LC, the highest weekly integral flux in E1 and E2 were detected simultaneously on MJD $=56973$ and were equal to $2.147 \pm 0.3 \times 10^{-6} \mathrm{phcm}^{-2} \mathrm{~s}^{-1}$ and $\quad 0.237 \pm 0.08 \times 10^{-6} \mathrm{phcm}^{-2} \mathrm{~s}^{-1}$, espectively.

The structural function $(S F)$ of the two flares for both energy ranges are shown in Figure (4) and Figure (5), respectively, binned in 12-hour intervals. The SF was used to study the characteristic time scale of the two flares. The SF for Flare I show indications for the time scale of 6,49 , and 55 days and 5.5, 24.5, 30, and 49 days for E1 and E2, respectively. It gives same time scale results for E1 and E2 around six and 49 days. There are no similarities between the general trends of the two flares for both energy ranges, that because flare II does not has obvious time scale in both energy ranges. The $A C F$ of the two flares for the two energy ranges are shown in Figure (6) and Figure (7), respectively, binned in 12-hour intervals. For Flare I the possible time scales were 6,49 , and 55 days (which are 
M.A. Hashad et al

interestingly identical to the corresponding SF results) and 25.5, and 49 days for E1 and E2, respectively. ACF results of flare I give same time scale for E1 and E2 around 49 days only. ACF for flare II does not give significant results.
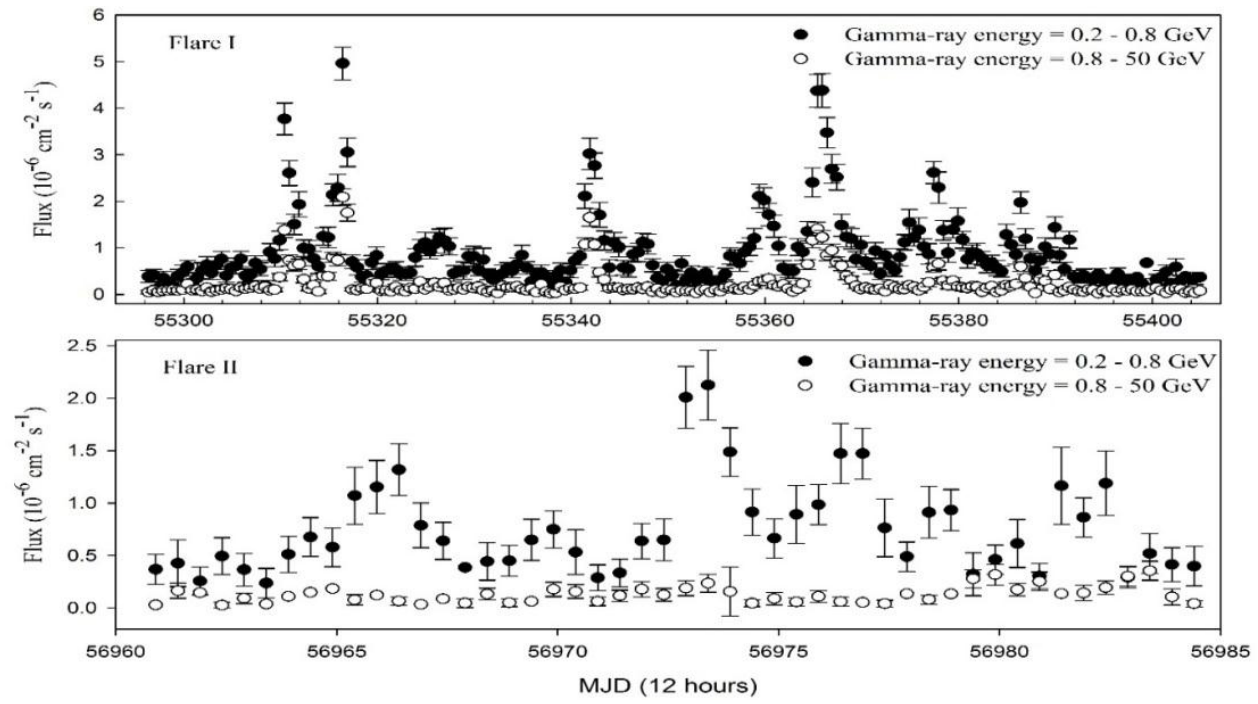

Figure (3) Top panel: High energy $\gamma$-ray light curves of $4 \mathrm{C}+21.35$ for $200-800 \mathrm{MeV}$ and $0.8-50 \mathrm{GeV}$ for flare I. Bottom panel: High energy $\gamma$-ray light curves of $4 \mathrm{C}+21.35$ for $200-800 \mathrm{MeV}$ and $0.8-50 \mathrm{GeV}$ for flare II. All data points are in 12-hour bin
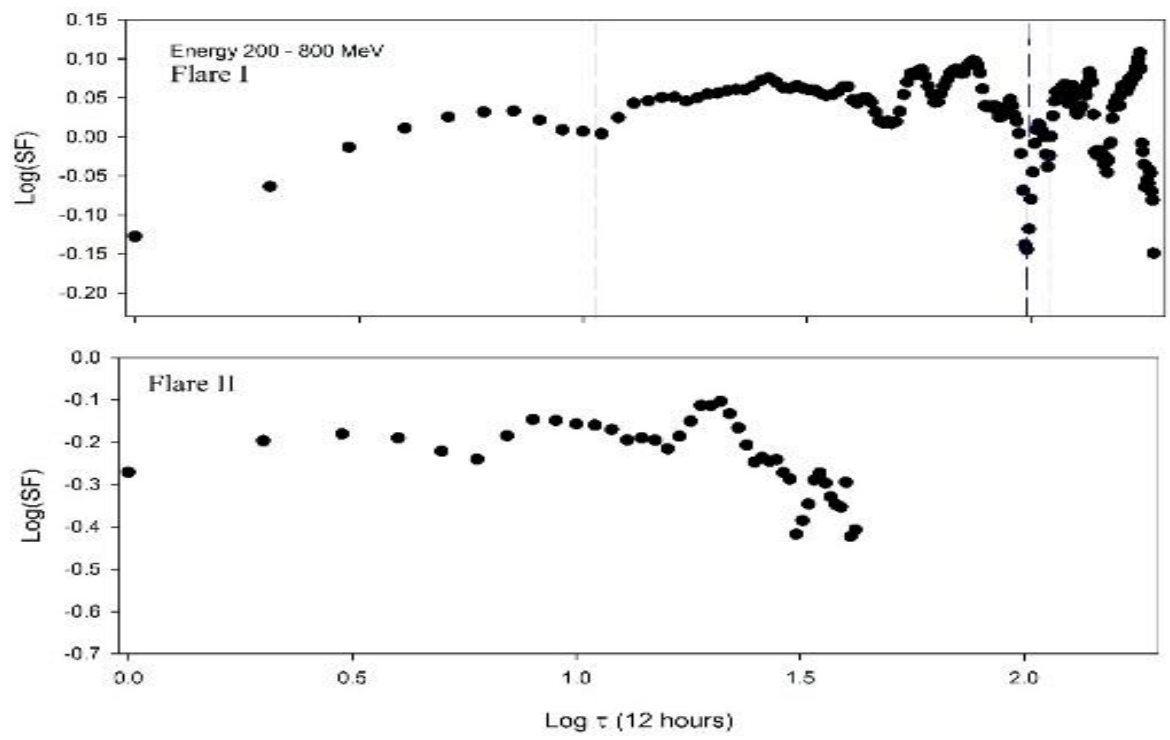

Figure (4) Structure functions of the two flares for 200-800 MeV gamma-ray energy 

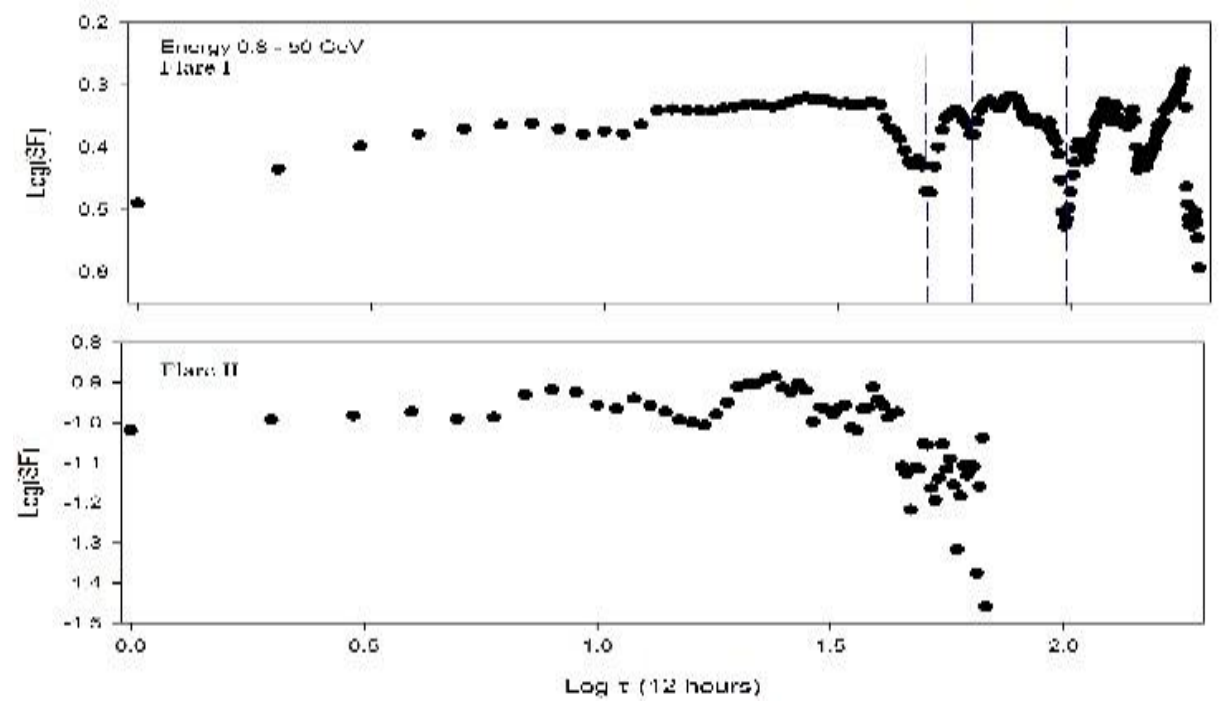

Figure (5) Structure functions of the two flares for $0.8-50 \mathrm{GeV}$ gamma-ray energy
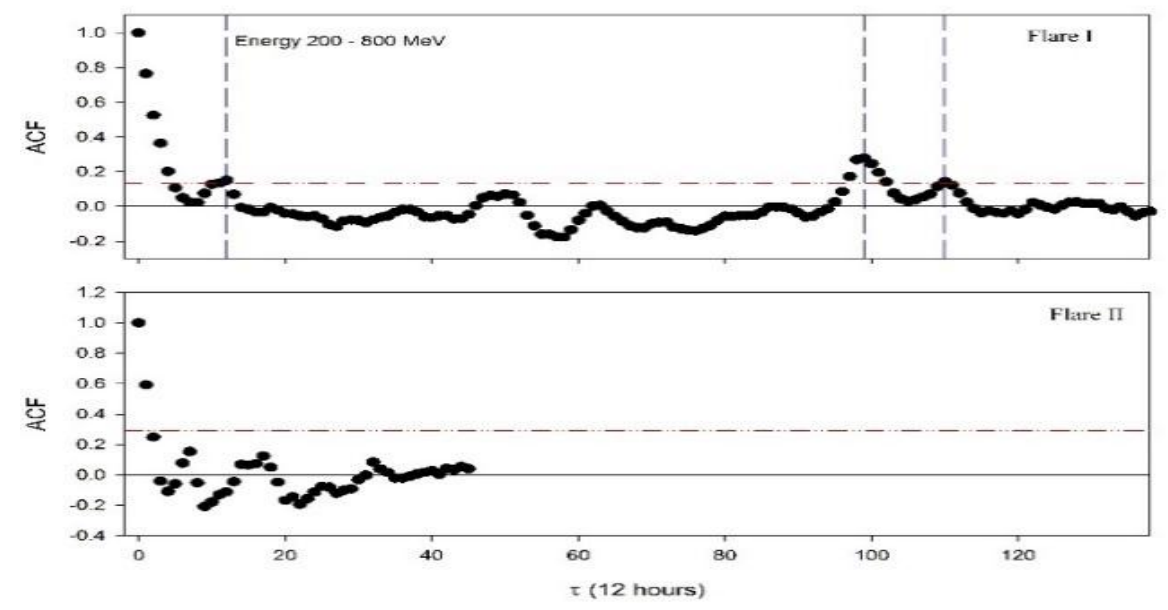

Figure (6) ACFs of the two flares for 200-800 MeV gamma-ray energy. The dashed dotted horizontal line refers to the confidence level
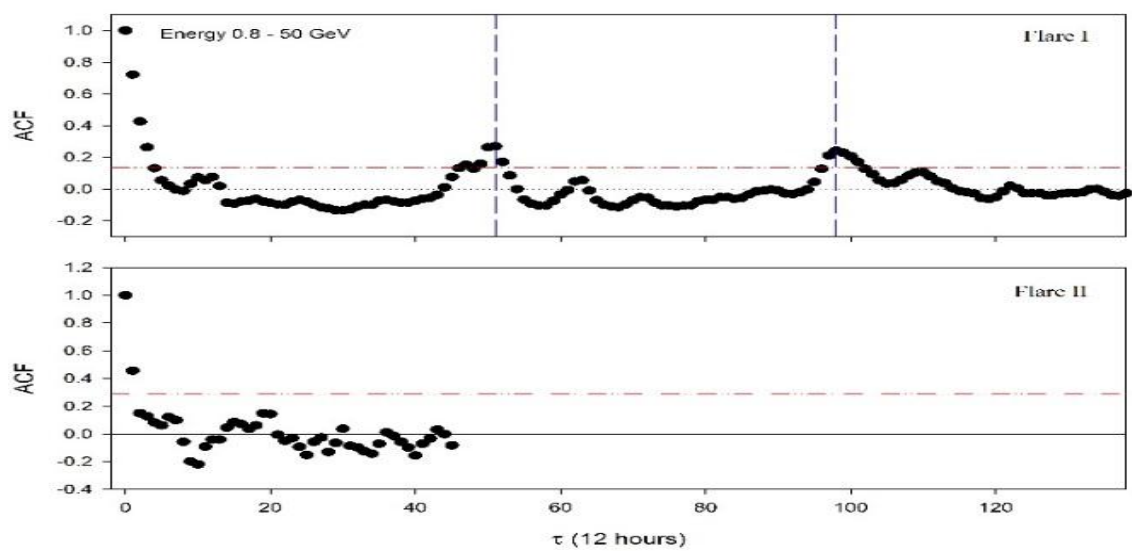

Figure (7) ACFs of the two flares for 0.8-50 GeV gamma-ray energy. The dashed dotted horizontal line refers to the confidence level 


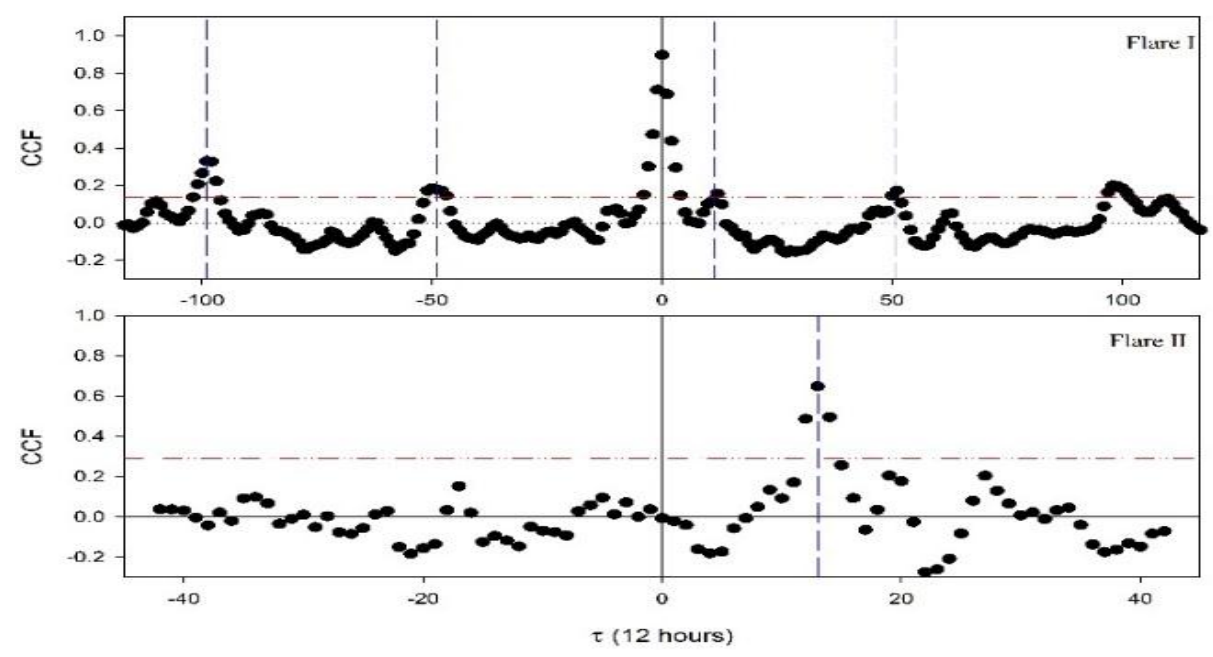

Figure (8) CCFs of the two flares between $200-800 \mathrm{MeV}$ and $0.8-50 \mathrm{GeV}$ gamma-ray energy bands. The dashed dotted horizontal line refers to the confidence level

\section{Conclusions}

A time study was performed for the light curve from FSRQ 4C 31+35 observed by the Fermi-LAT during the first seven years of the mission in energy range $0.2-50 \mathrm{GeV}$. The weekly light curve showed that the source reached the highest weekly integral flux $2.647 \times 10^{-6} \mathrm{phcm}^{-2} \mathrm{~s}^{-1}$ on MJD = 55365 (Jun. 18). This study reveals a large variety of temporal features and variability patterns in different energy bands $(0.2-0.8$ and $0.8-50$ $\mathrm{GeV})$. The variability of two brightest $\gamma$-ray flares episodes of this source was studied with 12-hour time binning in the two energy bands. The derived time scale obtained by SF analysis was crosschecked by ACF method. For the seven yearweekly binning light curve, SF illustrated typical values at the same time scales given in ACF, while the 18-week time scale, given one value, appears as a consequential from variability of Flare I. Flare I showed nearly same time scales from SF and ACF for both energy ranges, while Flare II did not yield any significant results. CCF between the two energy bands gave 0.0 and 6.5-day time lag for Flares I and II, respectively. Therefore, it can be extrapolated that the same emission mechanism is likely to be responsible for both E1 and E2 emission during Flare I. For Flare II, the emission mechanism of the low energy band (E1) came before the high energy one (E2) with 6.5 day with typical physical process.

Acknowledgments: Thanks are given to the anonymous referee for many suggestions that significantly improved the presentation of this study. This research is based on observations obtained with Fermi LAT science mission with instruments and contributions directly funded by Fermi LAT collaboration-NASA ${ }^{4}$. This work has been carried out using Enrico python script ${ }^{5}$.

\section{References}

1-Tanaka, Y. T., Stawarz, L., Thompson, D. J., et al. (2011) Fermi Large Telescope Detection of Bright $\gamma$ Ray Outburst from the Peculiar Quaser $4 \mathrm{C}+21.35$, The Astrophysical Journal, 733, 19-28.

2-Ghisellini, G., F. Tavecchio, L. Maraschi, A. Celotti, T. Sbarrato. (2014) The Power of Relativistic Jets is Larger than the Luminosity of their Accretion Disks, Nature, 515, 376-397.

3- Kushwaha, P., Singh, K. P., \& Sahayanathan, S. (2014) Brightest Fermi LAT Flares of PKS 1222+216: Implications on Emission and Acceleration Processes, The Astrophysical Journal, 796, 61-69.

4- Longo, F., Giroletti, M., Iafrate, G. (2009) Fermi LAT detection of Increasing Gamma-Ray Activity of Blazar PKS 1222+216, The Astronomer's Telegram, 2021, 1.

5-Verrecchia, F., Striani, E., Tavani, M., et al. (2009) AGILE Detection of a Gamma-Ray Flare from the Blazar PKS 1222+216, The Astronomer's Telegram, 2348, 1.

6- Ciprini, S. (2009) Fermi LAT confirmation of a strong GeV flare from 4C 21.35 (PKS 1222+21), The Astronomer's Telegram, 2349, 1. 
7-Dominici, T., Pereyra, A., et al. (2010) Optical polarimetric observations of $4 \mathrm{C}+21.35$ (PKS $1222+21$ ) during the gamma-ray flare, The Astronomer's Telegram, 2693, 1.

8- Abdo, A. A., et al. (2009) Early Fermi Gamma-ray Space Telescope Observations of the Quasar 3C 454.3, Astrophysics Journal, 699, 817.

9-Atwood, A.A., et al. (2009) The Large Area Telescope on the Fermi Gamma-Ray Space Telescope Mission, Astrophysical Journal, 697, 1071.

10-Sanchez, D. A., Deil. C. (2013) Enrico: A Python Package to Simplify Fermi-LAT Analysis, Proceedings of the 33rd International Cosmic Ray Conference (ICRC2013), Rio de Janeiro (Brazil), arXiv:1307.4534 [astro-ph.IM].

11-Badran, H. M., Abdou, Y., et al. (2016) Long-Term Time Variability of the TeV Blazer Mrk421 at HighEnergy Using Fermi-LAT Measurements, Isotope and Radiation Research 48, 171.
12- Simonetti, J. H., Cordes, J. M., \& Heeschen, D. S. (1985) Flicker of Extragalactic Radio Sources at Two Frequencies, The Astrophysical Journal, 296, 46.

13- Hughes, P. A., Aller, H. D., Aller, M. F. (1992) The University of Michigan Radio Astronomy Data Base. I. Structure Function Analysis and the Relation between BL Lacertae Objects and Quasi-stellar Objects, The Astrophysical Journal, 396, 469.

14- Edelson, R. A., Krolik, J. H. (1988) The Discrete Correlation Function: A New Method for Analyzing Unevenly Sampled Variability Data, The Astrophysical Journal, 333, 646.

15- Hufnagel, B. R., Bregman, J. N. (1992) Optical and Radio Variability in Blazars, The Astrophysical Journal, 386, 473.

16-Box, G. E. P., et al.1994 Time Series Analysis: Forecasting and Control, 3rd ed., Englewood Cliffs, NJ: Prentice Hall. 